\title{
The hook fusion procedure
}

\author{
James Grime \\ Department of Mathematics, University of York, York, YO10 5DD, UK \\ jrg112@york.ac.uk
}

\begin{abstract}
We derive a new expression for the diagonal matrix elements of irreducible representations of the symmetric group. We obtain this new expression using Cherednik's fusion procedure. However, instead of splitting Young diagrams into their rows or columns, we consider their principal hooks. This minimises the number of auxiliary parameters needed in the fusion procedure.
\end{abstract}

Submitted: Feb 25, 2005; Accepted: Apr 25, 2005; Published: Jun 3, 2005

Mathematics Subject Classifications: 05E10, 20C30

\section{Introduction}

In this article we present a new expression for the diagonal matrix elements of irreducible representations of the symmetric group. We will obtain this new expression using Cherednik's fusion procedure. This method originates from the work of Jucys [J], and has already been used by Nazarov and Tarasov [N1, NT]. However our approach differs by minimizing the number of auxiliary parameters needed in the fusion procedure. This is done by considering hooks of Young diagrams, rather than their rows or columns as in [N1, NT].

Irreducible representations of the group $S_{n}$ are parameterized by partitions of $n,[\mathrm{CR}]$. The Young diagram of a partition $\lambda$ is the set of boxes $(i, j) \in \mathbb{Z}^{2}$ such that $1 \leqslant j \leqslant \lambda_{i}$. In drawing such diagrams we let the first coordinate $i$ increase as one goes downwards, and the second coordinate $j$ increase from left to right. For example the partition $\lambda=(3,3,2)$ gives the diagram

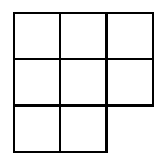

If $(i, j)$ is a box in the diagram of $\lambda$, then the $(i, j)$-hook is the set of boxes in $\lambda$

$$
\left\{\left(i, j^{\prime}\right): j^{\prime} \geqslant j\right\} \cup\left\{\left(i^{\prime}, j\right): i^{\prime} \geqslant i\right\}
$$

We call the $(i, i)$-hook the $i^{\text {th }}$ principal hook. 
A standard tableau, $\Lambda$, is a filling of the diagram $\lambda$ in which the entries are the numbers 1 to $n$, each occurring once. The Young Symmetrizer of a standard tableau $\Lambda$ of shape $\lambda$ generates an irreducible $\mathbb{C} S_{n}$-module, $[\mathrm{CR}]$, denoted $V_{\lambda}$.

In 1986 Ivan Cherednik proposed another description of the Young symmetrizer, [C]. The following version of Cherednik's description can be found in [NT].

There is a basis for the space $V_{\lambda}$ called the Young basis, with its vectors labeled by the standard tableaux of shape $\lambda$, [JK], [OV]. Now, for any standard tableau $\Lambda$ consider the diagonal matrix element, $F_{\Lambda}$, of the representation $V_{\lambda}$ corresponding to the Young basis vector $v_{\Lambda}$, defined by,

$$
F_{\Lambda}=\sum_{g \in S_{n}}\left(v_{\Lambda}, g v_{\Lambda}\right) g \in \mathbb{C} S_{n} .
$$

Multiplying $F_{\Lambda}$ on the left and right by certain invertible elements returns the Young symmetrizer. Hence $F_{\Lambda}$ may also be used to generate the irreducible module $V_{\lambda}$, [JK], [N3, section 2].

Cherednik's fusion procedure can be used to find an alternative expression for $F_{\Lambda}$. Consider a standard tableaux $\Lambda$ of shape $\lambda$. For each $p=1, \ldots, n$, put $c_{p}=j-i$ if the box $(i, j) \in \lambda$ is filled with the number $p$ in $\Lambda$. The difference $j-i$ is the content of box $(i, j)$.

For any two distinct numbers $p, q \in\{1, \ldots, n\}$, let $(p q)$ be the transposition in the symmetric group $S_{n}$. Consider the rational function of two complex variables $u$, $v$, with values in the group ring $\mathbb{C} S_{n}$ :

$$
f_{p q}(u, v)=1-\frac{(p q)}{u-v} .
$$

Now introduce $n$ complex variables $z_{1}, \ldots, z_{n}$. Consider the set of pairs $(p, q)$ with $1 \leqslant p<q \leqslant n$. Ordering the pairs lexicographically we form the product

$$
F_{\Lambda}\left(z_{1}, \ldots, z_{n}\right)=\prod_{(p, q)} f_{p q}\left(z_{p}+c_{p}, z_{q}+c_{q}\right) .
$$

If $p$ and $q$ sit on the same diagonal in the tableau $\Lambda$, then $f_{p q}\left(z_{p}+c_{p}, z_{q}+c_{q}\right)$ has a pole at $z_{p}=z_{q}$.

Let $\mathcal{R}_{\Lambda}$ be the vector subspace in $\mathbb{C}^{n}$ consisting of all tuples $\left(z_{1}, \ldots, z_{n}\right)$ such that $z_{p}=z_{q}$ whenever the numbers $p$ and $q$ appear in the same row of the tableau $\Lambda$.

By direct calculation we can show the following the identities are true;

$$
f_{p q}(u, v) f_{p r}(u, w) f_{q r}(v, w)=f_{q r}(v, w) f_{p r}(u, w) f_{p q}(u, v)
$$

for all pairwise distinct indices $p, q, r$, and

$$
f_{p q}(u, v) f_{s t}(z, w)=f_{s t}(z, w) f_{p q}(u, v)
$$


for all pairwise distinct $p, q, s, t$.

We call (3) and (4) the Yang-Baxter relations. Using these relations we obtain reduced expressions for $F_{\Lambda}\left(z_{1}, \ldots, z_{n}\right)$ different from the right hand side of (2). For more details on different expressions for $F_{\Lambda}\left(z_{1}, \ldots, z_{n}\right)$ see $[\mathrm{GP}]$.

Using (3) and (4) we may reorder the product $F_{\Lambda}\left(z_{1}, \ldots, z_{n}\right)$ such that each singularity is contained in an expression known to be regular at $z_{1}=z_{2}=\ldots=z_{n}$, [N2]. It is by this method that it was shown that the restriction of the rational function $F_{\Lambda}\left(z_{1}, \ldots, z_{n}\right)$ to the subspace $\mathcal{R}_{\Lambda}$ is regular at $z_{1}=z_{2}=\ldots=z_{n}$. Furthermore, by showing divisibility on the left and on the right by certain elements of $\mathbb{C} S_{n}$ it was shown that the value of $F_{\Lambda}\left(z_{1}, \ldots, z_{n}\right)$ at $z_{1}=z_{1}=\ldots=z_{n}$ is the diagonal matrix element $F_{\Lambda},[\mathrm{CR}]$. Thus we have the following theorem,

1.1 Restriction to $\mathcal{R}_{\Lambda}$ of the rational function $F_{\Lambda}\left(z_{1}, \ldots, z_{n}\right)$ is regular at $z_{1}=z_{2}=$ $\ldots=z_{n}$. The value of this restriction at $z_{1}=z_{2}=\ldots=z_{n}$ coincides with the element $F_{\Lambda} \in \mathbb{C} S_{n}$.

Similarly, we may form another expression for $F_{\Lambda}$ by considering the subspace in $\mathbb{C}^{n}$ consisting of all tuples $\left(z_{1}, \ldots, z_{n}\right)$ such that $z_{p}=z_{q}$ whenever the numbers $p$ and $q$ appear in the same column of the tableau $\Lambda[\mathrm{NT}]$.

In this article we present a new expression for the diagonal matrix elements which minimizes the number of auxiliary parameters needed in the fusion procedure. We do this by considering hooks of standard tableaux rather than their rows or columns.

Let $\mathcal{H}_{\Lambda}$ be the vector subspace in $\mathbb{C}^{n}$ consisting of all tuples $\left(z_{1}, \ldots, z_{n}\right)$ such that $z_{p}=z_{q}$ whenever the numbers $p$ and $q$ appear in the same principal hook of the tableau $\Lambda$. We will prove the following theorem.

Theorem 1.2 Restriction to $\mathcal{H}_{\Lambda}$ of the rational function $F_{\Lambda}\left(z_{1}, \ldots, z_{n}\right)$ is regular at $z_{1}=$ $z_{2}=\ldots=z_{n}$. The value of this restriction at $z_{1}=z_{2}=\ldots=z_{n}$ coincides with the element $F_{\Lambda} \in \mathbb{C} S_{n}$.

In particular, this hook fusion procedure can be used to form irreducible representations of $S_{n}$ corresponding to Young diagrams of hook shape using only one auxiliary parameter, $z$. By taking this parameter to be zero we find that no parameters are needed for diagrams of hook shape. Therefore if $\nu$ is a partition of hook shape we have

$$
F_{\nu}=F_{\nu}(z)=\prod_{(p, q)}^{\rightarrow} f_{p q}\left(c_{p}, c_{q}\right),
$$

with the pairs $(p, q)$ in the product ordered lexicographically.

To motivate the study of modules corresponding to partitions of hook shape first let us consider the Jacobi-Trudi identities [M, Chapter I3]. We can think of the following identities as dual to the Jacobi-Trudi identities. 
If $\lambda=\left(\lambda_{1}, \ldots, \lambda_{k}\right)$ such that $n=\lambda_{1}+\ldots+\lambda_{k}$ then we have the following decomposition of the induced representation of the tensor product of modules corresponding to the rows of $\lambda$;

$$
\operatorname{Ind}_{S_{\lambda_{1}} \times S_{\lambda_{2}} \times \cdots \times S_{\lambda_{k}}}^{S_{n}} V_{\left(\lambda_{1}\right)} \otimes V_{\left(\lambda_{2}\right)} \otimes \cdots \otimes V_{\left(\lambda_{k}\right)} \cong \bigoplus_{\mu}\left(V_{\mu}\right)^{\oplus K_{\mu \lambda}}
$$

where the sum is over all partitions of $n$. Note that $V_{\left(\lambda_{i}\right)}$ is the trivial representation of $S_{\lambda_{i}}$. The coefficients $K_{\mu \lambda}$ are non-negative integers known as Kostka numbers, [M]. Importantly, we have $K_{\lambda \lambda}=1$.

On the subspace $\mathcal{R}_{\Lambda}$, if $z_{i}-z_{j} \notin \mathbb{Z}$ when $i$ and $j$ are in different rows of $\Lambda$ then the above induced module may be realised as the left ideal in $\mathbb{C} S_{n}$ generated by $F_{\Lambda}\left(z_{1}, \ldots, z_{n}\right)$.

The irreducible representation $V_{\lambda}$ appears in the decomposition of this induced module with coefficient 1 , and is the ideal of $\mathbb{C} S_{n}$ generated by $F_{\Lambda}\left(z_{1}, \ldots, z_{n}\right)$ when $z_{1}=z_{2}=\ldots=$ $z_{n}$. The fusion procedure of theorem 1.1 provides a way of singling out this irreducible component.

Similarly we have the equivalent identity for columns,

$$
\operatorname{Ind}_{S_{\lambda_{1}^{\prime}} \times S_{\lambda_{2}^{\prime}} \times \cdots \times S_{\lambda_{l}^{\prime}}}^{S_{n}} V_{\left(1^{\lambda_{1}^{\prime}}\right)} \otimes V_{\left(1^{\lambda_{2}^{\prime}}\right)} \otimes \cdots \otimes V_{\left(1^{\lambda_{l}^{\prime}}\right)} \cong \bigoplus_{\mu}\left(V_{\mu}\right)^{\oplus K_{\mu^{\prime} \lambda^{\prime}}}
$$

where $l$ is the number of columns of $\lambda$. In this case $V_{\left(1^{\lambda_{i}^{\prime}}\right)}$ is the alternating representation of $S_{\lambda_{i}^{\prime}}$. This induced module is isomorphic to the left ideal of $\mathbb{C} S_{n}$ generated by $F_{\Lambda}\left(z_{1}, \ldots, z_{n}\right)$ considered on the subspace $\mathcal{R}_{\Lambda^{\prime}}$, with $z_{i}-z_{j} \notin \mathbb{Z}$ when $i, j$ are in different columns of $\Lambda$. Again the irreducible representation $V_{\lambda}$ appears in the decomposition of this induced module with coefficient 1 , and is the ideal of $\mathbb{C} S_{n}$ generated by $F_{\Lambda}\left(z_{1}, \ldots, z_{n}\right)$ when $z_{1}=z_{2}=\ldots=z_{n}$.

There is another expression known as the Giambelli identity [G]. Unlike the JacobiTrudi identities, this identity involves splitting $\lambda$ into its principal hooks, rather than its rows or columns. A combinatorial proof of the Giambelli identity can be found in [ER].

Divide a Young diagram $\lambda$ into boxes with positive and non-positive content. We may illustrate this on the Young diagram by drawing 'steps' above the main diagonal. Denote the boxes above the steps by $\alpha(\lambda)$ and the rest by $\beta(\lambda)$. For example, the following figure illustrates $\lambda, \alpha(\lambda)$ and $\beta(\lambda)$ for $\lambda=(3,3,2)$.
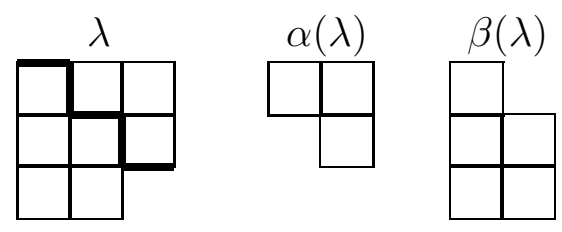

Figure 1: The Young diagram $(3,3,2)$ divided into boxes with positive content and nonpositive content 
If we denote the rows of $\alpha(\lambda)$ by $\alpha_{1}>\alpha_{2}>\ldots>\alpha_{d}>0$ and the columns of $\beta(\lambda)$ by $\beta_{1}>\beta_{2}>\ldots>\beta_{d}>0$, then we have the following alternative notation for $\lambda$;

$$
\lambda=(\alpha \mid \beta),
$$

where $\alpha=\left(\alpha_{1}, \ldots, \alpha_{d}\right)$ and $\beta=\left(\beta_{1}, \ldots, \beta_{d}\right)$.

Here $d$ denotes the length of the side of the Durfee square of shape $\lambda$, which is the set of boxes corresponding to the largest square that fits inside $\lambda$, and is equal to the number of principal hooks in $\lambda$. In our example $d=2$ and $\lambda=(2,1 \mid 3,2)$.

We may consider the following identity as a dual of the Giambelli identity.

$$
\operatorname{Ind}_{S_{h_{1}} S_{n}}^{S_{h_{2}} \times \cdots \times S_{h_{d}}} V_{\left(\alpha_{1} \mid \beta_{1}\right)} \otimes V_{\left(\alpha_{2} \mid \beta_{2}\right)} \otimes \cdots \otimes V_{\left(\alpha_{d} \mid \beta_{d}\right)} \cong \bigoplus_{\mu}\left(V_{\mu}\right)^{\oplus D_{\mu \lambda}}
$$

where $h_{i}$ is the length of the $i^{\text {th }}$ principal hook, and the sum is over all partitions of $n$. This is a decomposition of the induced representation of the tensor product of modules of hook shape. Further these hooks are the principal hooks of $\lambda$. The coefficients, $D_{\mu \lambda}$, are non-negative integers, and in particular $D_{\lambda \lambda}=1$.

On the subspace $\mathcal{H}_{\Lambda}$, if $z_{i}-z_{j} \notin \mathbb{Z}$ when $i$ and $j$ are in different principal hooks of $\Lambda$ then the above induced module may be realised as the left ideal in $\mathbb{C} S_{n}$ generated by $F_{\Lambda}\left(z_{1}, \ldots, z_{n}\right)$.

The irreducible representation $V_{\lambda}$ appears in the decomposition of this induced module with coefficient 1 , and is the ideal of $\mathbb{C} S_{n}$ generated by $F_{\Lambda}\left(z_{1}, \ldots, z_{n}\right)$ when $z_{1}=z_{2}=$ $\ldots=z_{n}$.

Hence, in this way, our hook fusion procedure relates to the Giambelli identity in the same way that Cherednik's original fusion procedure relates to the Jacobi-Trudi identity. Namely, it provides a way of singling out the irreducible component $V_{\lambda}$ from the above induced module.

The fusion procedure was originally developed in the study of affine Hecke algebras, $[\mathrm{C}]$. Our results may be regarded as an application of the representation theory of these algebras, $[\mathrm{OV}]$.

Acknowledgements and thanks go to Maxim Nazarov for his supervision, and for introducing me to this subject. I would also like to thank EPSRC for funding my research and my anonymous referees for their comments.

\section{Fusion Procedure for a Young Diagram}

The diagonal matrix element $F_{\Lambda}$ determines the irreducible module $V_{\lambda}$ of $S_{n}$, up to isomorphism, for any tableau $\Lambda$ of shape $\lambda$. Therefore in the sequel we will only use one particular tableau, the hook tableau. In which case we may denote the diagonal matrix element $F_{\Lambda}$ by $F_{\lambda}$, and the space $\mathcal{H}_{\Lambda}$ by $\mathcal{H}_{\lambda}$. 
We fill a diagram $\lambda$ by hooks to form a tableau $\Lambda$ in the following way: For the first principal hook we fill the column with entries $1,2, \ldots, r$ and then fill the row with entries $r+1, r+2, \ldots, s$. We then fill the column of the second principal hook with $s+1, s+2$, $\ldots, t$ and fill the row with $t+1, t+2, \ldots, x$. Continuing in this way we form the hook tableau.

Example 2.1. On the left is the hook tableau of the diagram $\lambda=(3,3,2)$, and on the right the same diagram with the content of each box.

\begin{tabular}{|l|l|l|}
\hline 1 & 4 & 5 \\
\hline 2 & 6 & 8 \\
\hline 3 & 7 & \multicolumn{1}{|c}{} \\
\cline { 1 - 2 } &
\end{tabular}

\begin{tabular}{|r|r|r|}
\hline 0 & 1 & 2 \\
\hline-1 & 0 & 1 \\
\hline-2 & -1 & \multicolumn{1}{|c}{} \\
\cline { 1 - 2 } &
\end{tabular}

Therefore the sequence $\left(c_{1}, c_{2}, \ldots, c_{8}\right)$ is given by $(0,-1,-2,1,2,0,-1,1)$.

Consider (2) as a rational function of the variables $z_{1}, \ldots, z_{n}$ with values in $\mathbb{C} S_{n}$. The factor $f_{p q}\left(z_{p}+c_{p}, z_{q}+c_{q}\right)$ has a pole at $z_{p}=z_{q}$ if and only if the numbers $p$ and $q$ stand on the same diagonal of the tableau $\Lambda$. We then call the pair $(p, q)$ a singularity. We call the corresponding term $f_{p q}\left(z_{p}+c_{p}, z_{q}+c_{q}\right)$ a singularity term, or simply a singularity.

Let $p$ and $q$ be in the same hook of $\Lambda$. If $p$ and $q$ are next to one another in the column of the hook then, on $\mathcal{H}_{\lambda}, f_{p q}\left(z_{p}+c_{p}, z_{q}+c_{q}\right)=1-(p q)$. So $\frac{1}{2} f_{p q}\left(z_{p}+c_{p}, z_{q}+c_{q}\right)$ is an idempotent. Similarly, if $p$ and $q$ are next to one another in the same row of the hook then $f_{p q}\left(z_{p}+c_{p}, z_{q}+c_{q}\right)=1+(p q)$, and $\frac{1}{2} f_{p q}\left(z_{p}+c_{p}, z_{q}+c_{q}\right)$ will be an idempotent.

Also, for distinct $p, q$, we have

$$
f_{p q}(u, v) f_{q p}(v, u)=1-\frac{1}{(u-v)^{2}} .
$$

Therefore, if the contents $c_{p}$ and $c_{q}$ differ by a number greater than one, then the factor $f_{p q}\left(z_{p}+c_{p}, z_{q}+c_{q}\right)$ is invertible in $\mathbb{C} S_{n}$ when $z_{p}=z_{q}$ with inverse $\frac{\left(c_{p}-c_{q}\right)^{2}}{\left(c_{p}-c_{q}\right)^{2}-1} f_{q p}\left(c_{q}, c_{p}\right)$.

The presence of singularity terms in the product $F_{\lambda}\left(z_{1}, \ldots, z_{n}\right)$ mean this product may or may not be regular on the vector subspace of $\mathcal{H}_{\lambda}$ consisting of all tuples $\left(z_{1}, \ldots, z_{n}\right)$ such that $z_{1}=z_{2}=\ldots=z_{n}$. Using the following lemma, we will be able to show that $F_{\lambda}\left(z_{1}, \ldots, z_{n}\right)$ is in fact regular on this subspace.

Lemma 2.2 The restriction of $f_{p q}(u, v) f_{p r}(u, w) f_{q r}(v, w)$ to the set $(u, v, w)$ such that $u=v \pm 1$ is regular at $u=w$.

Proof. Under the condition $u=v \pm 1$, the product $f_{p q}(u, v) f_{p r}(u, w) f_{q r}(v, w)$ can be written as

$$
(1 \mp(p q)) \cdot\left(1-\frac{(p r)+(q r)}{v-w}\right) .
$$

This rational function of $v, w$ is clearly regular at $w=v \pm 1$. 
Notice that the three term product, or triple, in the statement of the lemma can be written in reverse order due to (3). In particular, if the middle term is a singularity and the other two terms are an appropriate idempotent and triple term, then the triple is regular at $z_{1}=z_{2}=\ldots=z_{n}$. We may now prove the first statement of Theorem 1.2.

Proposition 2.3 The restriction of the rational function $F_{\lambda}\left(z_{1}, \ldots, z_{n}\right)$ to the subspace $\mathcal{H}_{\lambda}$ is regular at $z_{1}=z_{2}=\ldots=z_{n}$.

Proof. We will prove the statement by reordering the factors of the product $F_{\lambda}\left(z_{1}, \ldots, z_{n}\right)$, using relations (3) and (4), in such a way that each singularity is part of a triple which is regular at $z_{1}=z_{2}=\ldots=z_{n}$, and hence the whole of $F_{\lambda}\left(z_{1}, \ldots, z_{n}\right)$ will be manifestly regular.

Define $g_{p q}$ to be the following;

$$
g_{p q}=\left\{\begin{array}{cl}
f_{p q}\left(z_{p}+c_{p}, z_{q}+c_{q}\right) & \text { if } p<q \\
1 & \text { if } p \geqslant q
\end{array}\right.
$$

Now, let us divide the diagram $\lambda$ into two parts, consisting of those boxes with positive contents and those with non-positive contents as in Figure 1. Consider the entries of the $i^{\text {th }}$ column of the hook tableau $\Lambda$ of shape $\lambda$ that lie below the steps. If $u_{1}, u_{2}, \ldots, u_{k}$ are the entries of the $i^{\text {th }}$ column below the steps, we define

$$
C_{i}=\prod_{q=1}^{n} g_{u_{1}, q} g_{u_{2}, q} \ldots g_{u_{k}, q}
$$

Now consider the entries of the $i^{\text {th }}$ row of $\Lambda$ that lie above the steps. If $v_{1}, v_{2}, \ldots, v_{l}$ are the entries of the $i^{\text {th }}$ row above the steps, we define

$$
R_{i}=\prod_{q=1}^{n} g_{v_{1}, q} g_{v_{2}, q} \ldots g_{v_{l}, q}
$$

Our choice of the hook tableau was such that the following is true; if $d$ is the number of principal hooks of $\lambda$ then by relations (3) and (4) we may reorder the factors of $F_{\lambda}\left(z_{1}, \ldots, z_{n}\right)$ such that

$$
F_{\lambda}\left(z_{1}, \ldots, z_{n}\right)=\prod_{i=1}^{d} C_{i} R_{i} .
$$

Now, each singularity $(p, q)$ has its corresponding term $f_{p q}\left(z_{p}+c_{p}, z_{q}+c_{q}\right)$ contain in some product $C_{i}$ or $R_{i}$. This singularity term will be on the immediate left of the term $f_{p+1, q}\left(z_{p+1}+c_{p+1}, z_{q}+c_{q}\right)$. Also, this ordering has been chosen such that the product of factors to the left of any such singularity in $C_{i}$ or $R_{i}$ is divisible on the right by $f_{p, p+1}\left(z_{p}+c_{p}, z_{p+1}+c_{p+1}\right)$. 
Therefore we can replace the pair $f_{p q}\left(z_{p}+c_{p}, z_{q}+c_{q}\right) f_{p+1, q}\left(z_{p}+c_{p}, z_{q}+c_{q}\right)$ in the product by the triple $\frac{1}{2} f_{p, p+1}\left(z_{p}+c_{p}, z_{p+1}+c_{p+1}\right) f_{p q}\left(z_{p}+c_{p}, z_{q}+c_{q}\right) f_{p+1, q}\left(z_{p+1}+c_{p+1}, z_{q}+\right.$ $\left.c_{q}\right)$, where $\frac{1}{2} f_{p, p+1}\left(z_{p}+c_{p}, z_{p+1}+c_{p+1}\right)$ is an idempotent. Divisibility on the right by $f_{p, p+1}\left(z_{p}+c_{p}, z_{p+1}+c_{p+1}\right)$ means the addition of the idempotent has no effect on the value of the product $C_{i}$ or $R_{i}$.

By Lemma 2.2, the above triples are regular at $z_{1}=z_{2}=\ldots=z_{n}$, and therefore, so too are the products $C_{i}$ and $R_{i}$, for all $1 \leqslant i \leqslant d$. Moreover, this means $F_{\lambda}\left(z_{1}, \ldots, z_{n}\right)$ is regular at $z_{1}=z_{2}=\ldots=z_{n}$.

Example 2.4. As an example consider the hook tableau of the Young diagram $\lambda=(3,3,2)$, as shown in Example 2.1 .

In the original lexicographic ordering the product $F_{\lambda}\left(z_{1}, \ldots, z_{n}\right)$ is written as follows, for simplicity we will write $f_{p q}$ in place of the term $f_{p q}\left(z_{p}+c_{p}, z_{q}+c_{q}\right)$.

$$
\begin{aligned}
F_{\lambda}\left(z_{1}, \ldots, z_{n}\right)= & f_{12} f_{13} f_{14} f_{15} f_{16} f_{17} f_{18} f_{23} f_{24} f_{25} f_{26} f_{27} f_{28} f_{34} f_{35} f_{36} f_{37} f_{38} \\
& f_{45} f_{46} f_{47} f_{48} f_{56} f_{57} f_{58} f_{67} f_{68} f_{78}
\end{aligned}
$$

We may now reorder this product into the form below using relations (3) and (4) as described in the above proposition. The terms bracketed are the singularity terms with their appropriate triple terms.

$$
\begin{aligned}
F_{\lambda}\left(z_{1}, \ldots, z_{n}\right)= & f_{12} f_{13} f_{23} f_{14} f_{24} f_{34} f_{15} f_{25} f_{35}\left(f_{16} f_{26}\right) f_{36} f_{17}\left(f_{27} f_{37}\right) f_{18} f_{28} f_{38} \\
& \cdot f_{45} f_{46} f_{56} f_{47} f_{57}\left(f_{48} f_{58}\right) \cdot f_{67} f_{68} f_{78}
\end{aligned}
$$

So, for each singularity $f_{p q}$, we can replace $f_{p q} f_{p+1, q}$ by the triple $\frac{1}{2} f_{p, p+1} f_{p q} f_{p+1, q}$, where $\frac{1}{2} f_{p, p+1}$ is an idempotent, without changing the value of $F_{\lambda}\left(z_{1}, \ldots, z_{n}\right)$.

$$
\begin{aligned}
F_{\lambda}\left(z_{1}, \ldots, z_{n}\right)= & f_{12} f_{13} f_{23} f_{14} f_{24} f_{34} f_{15} f_{25} f_{35}\left(\frac{1}{2} f_{12} f_{16} f_{26}\right) f_{36} f_{17}\left(\frac{1}{2} f_{23} f_{27} f_{37}\right) f_{18} f_{28} f_{38} \\
& \cdot f_{45} f_{46} f_{56} f_{47} f_{57}\left(\frac{1}{2} f_{45} f_{48} f_{58}\right) \cdot f_{67} f_{68} f_{78}
\end{aligned}
$$

Since each of these triples is regular at $z_{1}=z_{2}=\ldots=z_{n}$ then so too is the whole of $F_{\lambda}\left(z_{1}, \ldots, z_{n}\right)$.

Therefore, due to the above proposition an element $F_{\lambda} \in \mathbb{C} S_{n}$ can now be defined as the value of $F_{\lambda}\left(z_{1}, \ldots, z_{n}\right)$ at $z_{1}=z_{2}=\ldots=z_{n}$. We proceed by showing this $F_{\lambda}$ is indeed the diagonal matrix element. To this end we will need the following propositions.

Note that for $n=1$, we have $F_{\lambda}=1$. For any $n \geqslant 1$, we have the following fact.

Proposition 2.5 The coefficient of $F_{\lambda} \in \mathbb{C} S_{n}$ at the unit element of $S_{n}$ is 1 .

Proof. For each $r=1, \ldots, n-1$ let $s_{r} \in S_{n}$ be the adjacent transposition $(r, r+1)$. Let $w_{0} \in S_{n}$ be the element of maximal length. Multiply the ordered product (2) by the 
element $w_{0}$ on the right. Using the reduced decomposition

$$
w_{0}=\prod_{(p, q)}^{\rightarrow} s_{q-p}
$$

we get the product

$$
\prod_{(p, q)}^{\rightarrow}\left(s_{q-p}-\frac{1}{z_{p}-z_{q}+c_{p}-c_{q}}\right) .
$$

For the derivation of this formula see $[\mathrm{GP},(2.4)]$. This formula expands as a sum of the elements $s \in S_{n}$ with coefficients from the field of rational functions of $z_{1}, \ldots, z_{n}$ valued in $\mathbb{C}$. Since the decomposition $(9)$ is reduced, the coefficient at $w_{0}$ is 1 . By the definition of $F_{\lambda}$, this implies that the coefficient of $F_{\lambda} w_{0} \in \mathbb{C} S_{n}$ at $w_{0}$ is also 1 .

In particular this shows that $F_{\lambda} \neq 0$ for any nonempty diagram $\lambda$. Let us now denote by $\varphi$ the involutive antiautomorphism of the group ring $\mathbb{C} S_{n}$ defined by $\varphi(g)=g^{-1}$ for every $g \in S_{n}$.

Proposition 2.6 The element $F_{\lambda} \in \mathbb{C} S_{n}$ is $\varphi$-invariant.

Proof. Any element of the group ring $\mathbb{C} S_{n}$ of the form $f_{p q}(u, v)$ is $\varphi$-invariant. Applying the antiautomorphism $\varphi$ to an element of the form (2) just reverses the ordering of the factors corresponding to the pairs $(p, q)$. However, the initial ordering can then be restored using relations (3) and (4), for more detail see [GP]. Therefore, any value of the function $F_{\lambda}\left(z_{1}, \ldots, z_{n}\right)$ is $\varphi$-invariant. Therefore, so too is the element $F_{\lambda}$.

Proposition 2.7 Let $x$ be last entry in the $k^{\text {th }}$ row of the hook tableau of shape $\lambda$. Denote by $\sigma_{x}$ the embedding $\mathbb{C} S_{n-x} \rightarrow \mathbb{C} S_{n}$ defined by $\sigma_{x}:(p q) \mapsto(p+x, q+x)$ for all distinct $p, q=1, \ldots, n-x$.

If $\lambda=\left(\alpha_{1}, \alpha_{2}, \ldots, \alpha_{d} \mid \beta_{1}, \beta_{2}, \ldots, \beta_{d}\right)$ and $\mu=\left(\alpha_{k+1}, \alpha_{k+2}, \ldots, \alpha_{d} \mid \beta_{k+1}, \beta_{k+2}, \ldots, \beta_{d}\right)$, then $F_{\lambda}=P \cdot \sigma_{x}\left(F_{\mu}\right)$, for some element $P \in \mathbb{C} S_{n}$.

Proof. Here the shape $\mu$ is obtained by removing the first $k$ principal hooks of $\lambda$. By the ordering described in Proposition 2.3,

$$
F_{\lambda}\left(z_{1}, \ldots, z_{n}\right)=\prod_{i=1}^{k} C_{i} R_{i} \cdot \sigma_{x}\left(F_{\mu}\left(z_{x+1}, \ldots, z_{n}\right)\right),
$$

where $C_{i}$ and $R_{i}$ are defined by (7) and (8).

Since all products $C_{i}$ and $R_{i}$ are regular at $z_{1}=z_{2}=\ldots=z_{n}$, Proposition 2.3 then gives us the required statement. 
In any given ordering of $F_{\lambda}\left(z_{1}, \ldots, z_{n}\right)$, we want a singularity term to be placed next to an appropriate triple term such that we may then form a regular triple. In that case we will say these two terms are 'tied'.

We now complete the proof of Theorem 1.2. If $u, v$ stand next to each other in the same row, or same column, of $\Lambda$ the following results show that $F_{\lambda}$ is divisible on the left (and on the right) by $1-(u v)$, or $1+(u v)$ respectively, or divisible by these terms preceded (followed) by some invertible elements of $\mathbb{C} S_{n}$. Hence $F_{\lambda}$ is the diagonal matrix element.

However, proving the divisibilities described requires some pairs to be 'untied', in which case we must form a new ordering. This is the content of the following proofs. Some explicit examples will then given in Example 2.10 below.

Proposition 2.8 Suppose the numbers $u<v$ stand next to each other in the same column of the hook tableau $\Lambda$ of shape $\lambda$. First, let $s$ be the last entry in the row containing $u$. If $c_{v}<0$ then the element $F_{\lambda} \in \mathbb{C} S_{n}$ is divisible on the left and on the right by $f_{u, v}\left(c_{u}, c_{v}\right)=1-(u v)$. If $c_{v} \geqslant 0$ then the element $F_{\lambda} \in \mathbb{C} S_{n}$ is divisible on the left by the product

$$
\prod_{i=u, \ldots, s}^{\leftarrow}\left(\prod_{j=s+1, \ldots, v} f_{i j}\left(c_{i}, c_{j}\right)\right)
$$

Proof. By Proposition 2.6, the divisibility of $F_{\lambda}$ by the element $1-(u v)$ on the left is equivalent to the divisibility by the same element on the right. Let us prove divisibility on the left.

By Proposition 2.7, if $\sigma_{x}\left(F_{\mu}\right)$ is divisible on the right by $f_{u v}\left(c_{u}, c_{v}\right)$, or $f_{u v}\left(c_{u}, c_{v}\right)$ followed by some invertible terms, then so too will $F_{\lambda}$. If $\sigma_{x}\left(F_{\mu}\right)$ is divisible on the left by $f_{u v}\left(c_{u}, c_{v}\right)$, or $f_{u v}\left(c_{u}, c_{v}\right)$ preceded by some invertible terms, then, by using Proposition 2.6 twice, first on $\sigma_{x}\left(F_{\mu}\right)$ then on the product $F_{\lambda}=P \cdot \sigma_{x}\left(F_{\mu}\right)$, so too will $F_{\lambda}$. Hence we only need to prove the statement for $(u, v)$ such that $u$ is in the first row or first column of $\Lambda$.

Let $r$ be the last entry in the first column of $\Lambda, s$ the last entry in the first row of $\Lambda$, and $t$ the last entry in the second column of $\Lambda$, as shown in Figure 2.

We now continue this proof by considering three cases and showing the appropriate divisibility in each.

(i) If $c_{v}<0$ (i.e. $u$ and $v$ are in the first column of $\Lambda$ ) then $v=u+1$ and $F_{\lambda}\left(z_{1}, \ldots, z_{n}\right)$ can be written as $F_{\lambda}\left(z_{1}, \ldots, z_{n}\right)=f_{u v}\left(z_{u}+c_{u}, z_{v}+c_{v}\right) \cdot F$.

Starting with $F_{\lambda}\left(z_{1}, \ldots, z_{n}\right)$ written in the ordering described in Proposition 2.3 and simply moving the term $f_{u, v}\left(z_{u}+c_{u}, z_{v}+c_{v}\right)$ to the left results in all the singularity terms in the product $F$ remaining tied to the same triple terms as originally described in that ordering. Therefore we may still form regular triples for each singularity in $F$, and hence $F$ is regular at $z_{1}=z_{2}=\ldots=z_{n}$. 


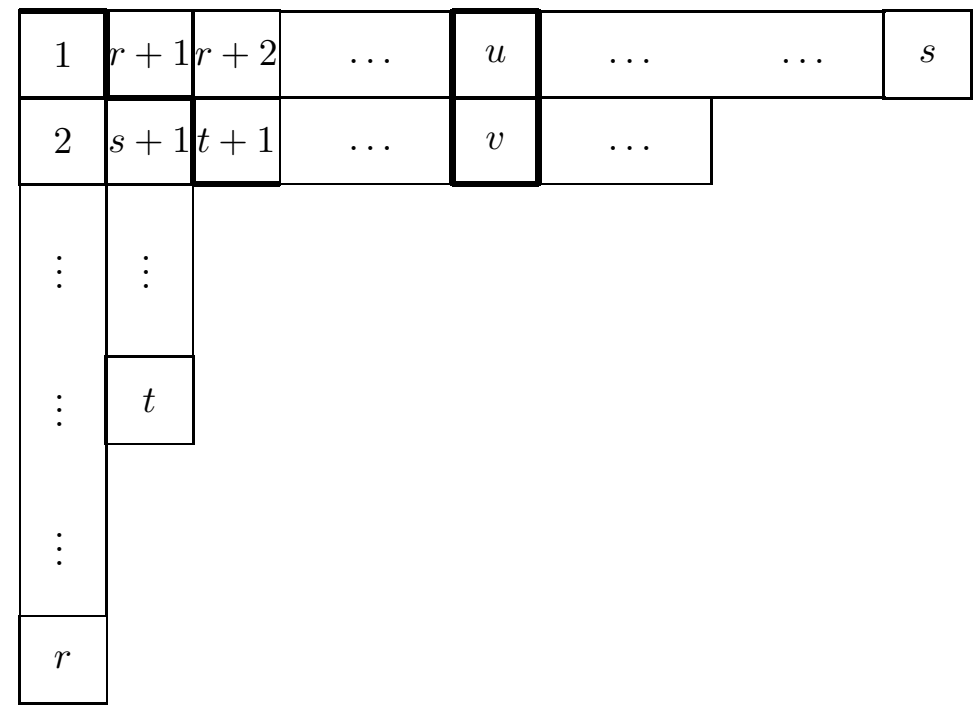

Figure 2: The first two principal hooks of the hook tableau $\Lambda$

So by considering this expression for $F_{\lambda}\left(z_{1}, \ldots, z_{n}\right)$ at $z_{1}=z_{2}=\ldots=z_{n}$ we see that $F_{\lambda}$ will be divisible on the left/right by $f_{u v}\left(c_{u}, c_{v}\right)=(1-(u v))$.

(ii) If $c_{v}=0$ then $v=s+1$, and $F_{\lambda}\left(z_{1}, \ldots, z_{n}\right)$ can be written as

$$
F_{\lambda}\left(z_{1}, \ldots, z_{n}\right)=\prod_{i=u, \ldots, s}^{\leftarrow} f_{i, s+1}\left(z_{i}+c_{i}, z_{s+1}+c_{s+1}\right) \cdot F^{\prime}
$$

Again, starting with the ordering described in Proposition 2.3, this results in all the singularity terms in the product $F^{\prime}$ remaining tied to the same triple terms as originally described in that ordering. Hence $F^{\prime}$ is regular at $z_{1}=z_{2}=\ldots=z_{n}$. So $F_{\lambda}$ is divisible on the left by

$$
\prod_{i=u, \ldots, s}^{\leftarrow} f_{i, s+1}\left(c_{i}, c_{s+1}\right)
$$

(iii) If $c_{v}>0$ (i.e. $v$ is above the steps) then $f_{u v}\left(z_{u}+c_{u}, z_{v}+c_{v}\right)$ is tied to the singularity $f_{u-1, v}\left(z_{u-1}+c_{u-1}, z_{v}+c_{v}\right)$ as a triple term. To show divisibility by $f_{u v}\left(z_{u}+\right.$ $\left.c_{u}, z_{v}+c_{v}\right)$ in this case we need an alternative expression for $F_{\lambda}\left(z_{1}, \ldots, z_{n}\right)$ that is regular when $z_{1}=z_{2}=\ldots=z_{n}$. Define a permutation $\tau$ as follows,

$$
\begin{aligned}
& \tau=\prod_{i=u, \ldots, s}^{\rightarrow}\left(\prod_{j=s+1, \ldots, v}^{\leftarrow}(i j)\right)
\end{aligned}
$$

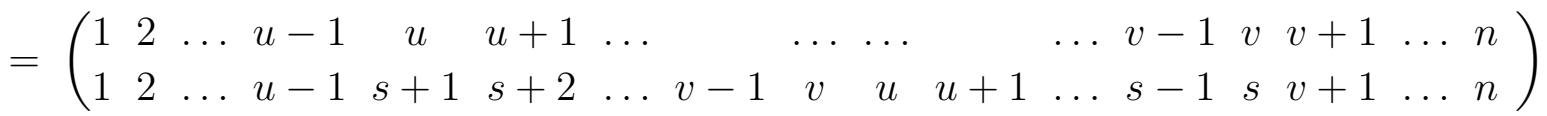


From the definition of $C_{1}$ in (7) we now define $C_{1}^{\prime}=\tau C_{1}$, where $\tau$ acts on the indices of the product $C_{1}$ such that

$$
\tau C_{1}=\prod_{j=1}^{n}\left(\prod_{i=1}^{r} g_{i, \tau \cdot j}\right)
$$

For the rest of this proof we will simply write $f_{i j}$ instead of $f_{i j}\left(z_{i}+c_{i}, z_{j}+c_{j}\right)$. Define $R_{1}^{\prime}$ as,

$$
\begin{aligned}
& R_{1}^{\prime}=\prod_{i=r+2, \ldots, u-1}^{\leftarrow}\left(\prod_{j=s+1, \ldots, v} f_{i j}\right) \cdot \prod_{i=s+1, \ldots, t-1}\left(\prod_{j=i+1, \ldots, t}^{\rightarrow} f_{i j}\right) \cdot\left(\prod_{j=s+1, \ldots, t}^{\leftarrow} f_{r+1, j}\right) \\
& \times\left(\prod_{j=t+1, \ldots, v} f_{r+1, j}\right) \prod_{i=r+1, \ldots, s-1}\left(\prod_{j=i+1, \ldots, s} f_{i j}\right) \cdot \prod_{j=v+1, \ldots, n}\left(\prod_{i=r+1, \ldots, s} f_{i j}\right) .
\end{aligned}
$$

Finally, define $C_{2}^{\prime}$ as,

$$
C_{2}^{\prime}=\prod_{j=t+1, \ldots, n}^{\rightarrow}\left(\prod_{i=s+1, \ldots, t} f_{i j}\right)
$$

Then,

$$
F_{\lambda}\left(z_{1}, \ldots, z_{n}\right)=\prod_{i=u, \ldots, s}^{\leftarrow}\left(\prod_{j=s+1, \ldots, v}^{\rightarrow} f_{i j}\right) \cdot C_{1}^{\prime} R_{1}^{\prime} C_{2}^{\prime} R_{2} \cdot \prod_{i=3}^{d} C_{i} R_{i}
$$

where $d$ is the number of principal hooks of $\lambda$.

The product $C_{1}^{\prime} R_{1}^{\prime} C_{2}^{\prime} R_{2} \cdot \prod C_{i} R_{i}$ is regular at $z_{1}=z_{2}=\ldots z_{n}$ since, as before, for any singularity $(p, q)$ the terms $f_{p q} f_{p+1, q}$ can be replaced by the triple $\frac{1}{2} f_{p, p+1} f_{p q} f_{p+1, q}-$ except in the expression $R_{1}^{\prime}$ where the terms $f_{p l} f_{p q}$ are replaced by $\frac{1}{2} f_{p l} f_{p q} f_{l q}$, where $l$ is the entry to the immediate left of $q$. Note that $l=q-1$ when $c_{q}>1$ and $l=s+1$ when $c_{q}=1$.

So by letting $z_{1}=z_{2}=\ldots=z_{n}$ we see that $F_{\lambda}$ is divisible on the left by

$$
\prod_{i=u, \ldots, s}^{\leftarrow}\left(\prod_{j=s+1, \ldots, v} f_{i j}\left(c_{i}, c_{j}\right)\right)
$$

Proposition 2.9 Suppose the numbers $u<v$ stand next to each other in the same row of the hook tableau $\Lambda$ of shape $\lambda$. Let $r$ be the last entry in the column containing $u$. If $c_{u}>0$ then the element $F_{\lambda} \in \mathbb{C} S_{n}$ is divisible on the left and on the right by $f_{u, v}\left(c_{u}, c_{v}\right)=1+(u v)$. If $c_{u} \leqslant 0$ then the element $F_{\lambda} \in \mathbb{C} S_{n}$ is divisible on the left by the product

$$
\prod_{i=u, \ldots, r}^{\leftarrow}\left(\prod_{j=r+1, \ldots, v}^{\rightarrow} f_{i j}\left(c_{i}, c_{j}\right)\right)
$$


We omit the proof of this proposition as it is very similar to that of Proposition 2.8.

This completes our proof of Theorem 1.2. Let us now consider an example that allows us to see how the product $F_{\lambda}\left(z_{1}, \ldots, z_{n}\right)$ is broken down in the proof of Proposition 2.8.

Example 2.10. We again consider the hook tableau of the Young diagram $\lambda=$ $(3,3,2)$, as shown in Example 2.1 .

We begin with the product $F_{\lambda}\left(z_{1}, \ldots, z_{n}\right)$ in the ordering described in Proposition 2.3. For simplicity we again write $f_{p q}$ in place of the term $f_{p q}\left(z_{p}+c_{p}, z_{q}+c_{q}\right)$. We have also marked out the singularities in this expansion along with their triple terms, but no idempotents have yet been added which would form regular triples.

$$
\begin{aligned}
F_{\lambda}\left(z_{1}, \ldots, z_{n}\right)= & f_{12} f_{13} f_{23} f_{14} f_{24} f_{34} f_{15} f_{25} f_{35}\left(f_{16} f_{26}\right) f_{36} f_{17}\left(f_{27} f_{37}\right) f_{18} f_{28} f_{38} \\
& \cdot f_{45} f_{46} f_{56} f_{47} f_{57}\left(f_{48} f_{58}\right) \cdot f_{67} f_{68} f_{78}
\end{aligned}
$$

Let $u=4$ and $v=6$ in Proposition 2.8. Then by that proposition we may arrange the above product as follows. Notice since $c_{v}=0$ all singularity-triple term pairs remain the same.

$$
\begin{aligned}
F_{\lambda}\left(z_{1}, \ldots, z_{n}\right)= & f_{56} f_{46} \cdot f_{12} f_{13} f_{23}\left(f_{16} f_{26}\right) f_{36} f_{14} f_{24} f_{34} f_{15} f_{25} f_{35} f_{17}\left(f_{27} f_{37}\right) \\
& f_{18} f_{28} f_{38} \cdot f_{45} f_{47} f_{57}\left(f_{48} f_{58}\right) \cdot f_{67} f_{68} f_{78}
\end{aligned}
$$

We may now add the appropriate idempotents so that all singularities remain in regular triples. So by considering the product at $z_{1}=z_{2}=\ldots=z_{n}$ we have that $F_{\lambda}$ is divisible on the left by $(1-(46))$, preceded only by invertible terms, as desired.

Now let $u=5$ and $v=8$. As described by Proposition 2.8 (iii) we may arrange (10) in the following way. Singularities in $R_{1}$ have been marked out with their alternative triple terms, while all other singularity-triple term pairs remain the same.

$$
\begin{aligned}
F_{\lambda}\left(z_{1}, \ldots, z_{n}\right)= & f_{56} f_{57} f_{58} \cdot f_{12} f_{13} f_{23} f_{14} f_{24} f_{34}\left(f_{16} f_{26}\right) f_{36} f_{17}\left(f_{27} f_{37}\right) f_{18} f_{28} f_{38} \\
& f_{15} f_{25} f_{35} \cdot f_{67} f_{47}\left(f_{46} f_{48}\right) f_{45} \cdot f_{68} f_{78}
\end{aligned}
$$

In moving $f_{58}$ to the left it is untied from the singularity $f_{48}$. So we must form new triples which are regular at $z_{1}=z_{2}=\ldots=z_{n}$. Therefore, by considering the product at $z_{1}=z_{2}=\ldots=z_{n}$, we have that $F_{\lambda}$ is divisible on the left by $(1-(58))$, again preceded only by invertible terms, as desired.

\section{References}

[C] I. V. Cherednik, On special bases of irreducible finite-dimensional representations of the degenerate affine Hecke algebra, Funct. Anal. Appl. 20 (1986) 87-89.

[CR] C. Curtis and I. Reiner, Representation theory of finite groups and associative algebras, Interscience Publishers, 1962. 
[ER] Ö. N. Eğecioğlu and J.B. Remmel, A combinatorial proof of the Giambelli Identity for Schur functions, Adv. in Math. 70 (1988), no. 1, 59-86.

[G] G. Z. Giambelli, Alcuna propreita delle funzioni simmetriche charatteristiche, Atti Accad. Sci. Torino Cl. Sci. Fis. Mat. Natur. 38 (1903), 323-344.

[GP] V. Guizi and P. Papi, A combinatorial approach to the fusion process for the symmetric group, European Journal of Combinatorics, 19 (1998), 835-845.

[J] A. Jucys, On the Young operators of the symmetric group, Lietuvos Fizikos Rinkinys. 6 (1966), 163-180.

[JK] G. James and A. Kerber, The representation theory of the symmetric group, Encyclopedia of mathematics and its applications 16 (1981).

[M] I. G. Macdonald, Symmetric functions and Hall polynomials, Oxford Univ. Press, London/New York, 1979.

[N1] M. Nazarov, Young's symmetrizers for projective representations of the symmetric group, Adv. Math. 127 (1997), no. 2, 190-257.

[N2] M. Nazarov, Yangians and Capelli Identities, Amer. Math. Soc Translations 181 (1998), 139-163.

[N3] M. Nazarov, Representations of twisted Yangians associated with skew Young diagrams, Selecta Math. 10 (2004), 71-129.

[NT] M. Nazarov and V. Tarasov, On irreducibility of tensor products of Yangian modules associated with skew Young diagrams., Duke Math Jour. 112 (2002), 343-378.

[OV] A. Okounkov and A. Vershik, A new approach to representation theory of symmetric groups, Selecta Math, 2 (1996), no. 4, 581-605. 